\title{
Covalent Immobilization of Peptides on Self-Assembled Monolayer Surfaces using Soft-Landing of Mass-Selected Ions
}

Peng Wang, Omar Hadjar, and Julia Laskin

\section{Experimental}

Ion Soft-landing

SL experiments were performed in the newly designed and constructed softlanding apparatus described in details elsewhere. ${ }^{1}$ Briefly, protonated peptide ions are produced in an electrospray ionization (ESI) source and introduced into the vacuum system using an electrodynamic ion funnel. After the ion funnel, ions undergo collisional relaxation in a collisional quadrupole (CQ) followed by mass selection using a commercial Extrel (Pittsburgh, PA) quadrupole mass filter (resolving quadrupole, RQ). Mass-selected ions are transmitted through a series of einzel lenses that enable precise positioning and shaping of the ion beam. An electrostatic quadrupole bender located after the second set of enzel lens turns the ion beam by $90^{\circ}$ to avoid contamination of the surface by neutrals. After exiting the last set of enzel lens, mass-selected ions are decelerated between two meshes and collide with the surface at specific energies. Collision energy of the projectile ions is defined as the potential difference between the $\mathrm{CQ}$ and the surface multiplied by the charge state of the ions. By biasing the surface at different voltage, ions can be deposited with various energies. A background pressure of $2 \times 10^{-9}$ is maintained in the deposition chamber. Surfaces can be introduced or removed through a vacuum interlock system. Depending on the properties of different peptides, ion currents ranging from a few $\mathrm{pA}$ to $60 \mathrm{pA}$ are achieved at the target. And the beam size is tunable from $1.5 \mathrm{~mm}$ to $6 \mathrm{~mm}$. For IRRAS characterization, we positioned the target right after the resolving quardrupole; typical ion current of $400 \mathrm{pA}$ was measured on the target. Using $0.1 \mathrm{mM} \mathrm{c(-RGDfK-)} \mathrm{solution} \mathrm{in} \mathrm{methanol} \mathrm{and} 15 \mu \mathrm{L} / \mathrm{h}$ flow rate, we were able to deliver ca. monolayer of c(-RGDfK-) onto a $5 \mathrm{~mm}$ spot in $4 \mathrm{hrs}$, which corresponds to a consumption of $6 \mathrm{nmol}$ of c(-RGDfK-). In contrast, the solution-phase experiment utilized at least $1 \mathrm{~mL}$ of $2 \mathrm{mM}$ c(-RGDfK-) solution corresponding to $2 \mu \mathrm{mol}$ of c(-RGDfK-). We estimate that the solution-phase experiment required two orders of magnitude more material than the SL experiment. 
Self-Assembled Monolayer (SAM) Surface

Dithiobis (succinimidyl undecanoate) (Dojindo Laboratories) was used to generate N-hydroxylsuccinimidyl ester terminated SAM. The SAMs were prepared following literature procedures. ${ }^{2} 10 \times 10 \mathrm{~mm}$ gold coated silicon wafers with $100 \mathrm{~nm}$ gold layer deposited on top of a $10 \mathrm{~nm}$ chromium adhesion layer (SPI Supplies) were thoroughly cleaned in an ultraviolet/ozone cleaner before the deposition of the SAMs. The gold substrates were then immersed in $1 \mathrm{mM}$ dithiobis solution in ethanol $(20 \% \mathrm{v} / \mathrm{v}$ methylene dicholoride was added to increase the solubility of dithiobis) at room temperature for $12 \mathrm{hrs}$. After the growth process, the samples were removed from the thiol solution followed by rigorously cleaning in pure ethanol with an ultrasonic bath to remove physisorbed molecules from the SAM surface. Finally the SAMs were dried in a pure nitrogen stream.

1) Hadjar, O.; Wang, P; Futrell, J. H.; Dessiaterik, Y.; Zhu, Z.; Cowin, J. P.; Iedema, M. J.; Laskin, J. submitted to Anal. Chem

2) Wagner, P.; Hegner, M.; Kernen, P.; Zaugg, F.; Semenza, G. Biophys. J. 1996, 70, 2052 


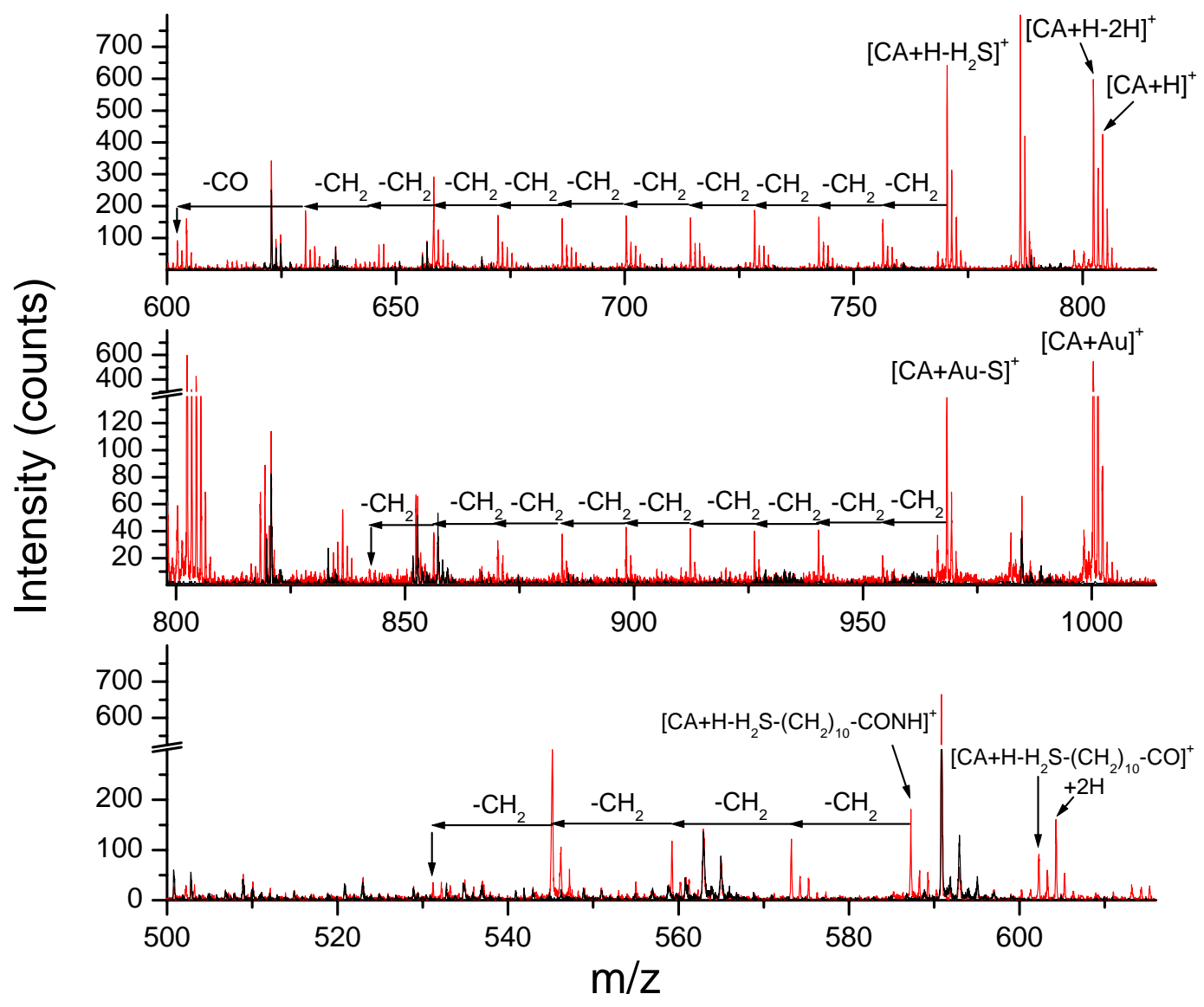

Figure S1. ToF-SIMS spectrum of the NHS-SAM surface following $2 \mathrm{hr}$ SL of c(RGDfK-) and extensive rinsing (red) as shown in Figure 1c with detailed peak assignments and the unmodified NHS-SAM (black). Top panel shows the protonated doublet at $\mathrm{m} / \mathrm{z} 804.4$ and 802.4 of the covalent adduct (CA) and the related fragments; middle panel shows the CA cationized on gold and the related fragments; bottom panel shows lower-mass part of the spectrum that contains characteristic peaks corresponding to the loss of NHCO from the amide bond between c(-RGDfK-) and the SAM chain and loss of up to four $-\mathrm{CH}_{2}$ moieties from the lysine side chain of c(-RGDfK-).

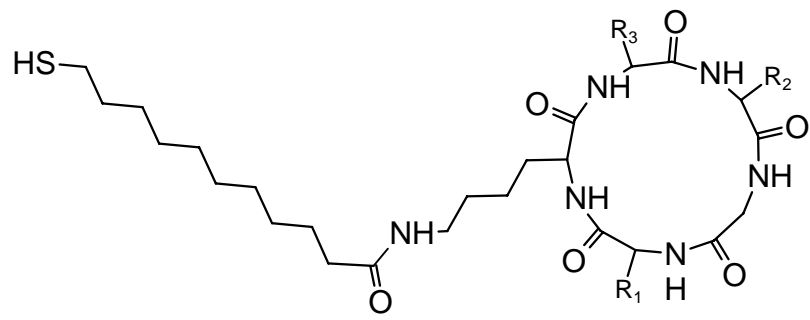

Schematic drawing of the structure of the covalent adduct (CA). 